\title{
Research Article \\ Quantization of Free Scalar Fields in the Presence of Natural Cutoffs
}

\author{
K. Nozari, F. Moafi, and F. Rezaee Balef \\ Department of Physics, Islamic Azad University, Sari Branch, Sari, Iran \\ Correspondence should be addressed to K. Nozari, knozari@umz.ac.ir
}

Received 22 September 2012; Accepted 29 October 2012

Academic Editor: Joseph Formaggio

Copyright $@ 2012$ K. Nozari et al. This is an open access article distributed under the Creative Commons Attribution License, which permits unrestricted use, distribution, and reproduction in any medium, provided the original work is properly cited.

We construct a quantum theory of free scalar fields in $(1+1)$-dimensions based on the deformed Heisenberg algebra $[\widehat{x}, \hat{p}]=i \hbar\left(1-\beta p+2 \beta^{2} p^{2}\right)$, that admits the existence of both a minimal measurable length and a maximal momentum, where $\beta$ is a deformation parameter. We consider both canonical and path integral formalisms of the scenario. Finally a higher dimensional extension is easily performed in the path integral formalism.

\section{Introduction}

Physics in extremely high energy scales is particularly of interest to particle physicists. It is now a well-known issue that gravity induces uncertainty in measurement of physical quantities. By now, string theory is one of the most successful theoretical frameworks which overcomes the difficulty of ultraviolet divergences in quantum theory of gravity. Incorporation of gravity in quantum field theory leads naturally to an effective cutoff (a minimal measurable length) in the ultraviolet regime. Therefore, if we construct a field theory which captures some stringy nature and/or includes stringy corrections, then it would play a crucial role in investigation of physics at high energy scales towards the Planck scale. Some of the stringy corrections appear as higher order polynomials of momentum leading to modified dispersion relations (see e.g., [1] and references therein). One of the stringy corrections is deforming the Heisenberg uncertainty principle to a generalized uncertainty principle (GUP). Some approaches to quantum gravity such as string theory [2-9], loop quantum gravity [10], and quantum geometry [11-13] all indicated the existence of a minimal measurable length of the order of the Planck length, $l_{\mathrm{pl}} \sim 10^{-35} \mathrm{~m}$ (see also [14-17]). Moreover some Gedanken experiments in the spirit of black hole physics have also supported the idea of existence of a minimal measurable length [18]. So, existence of a minimal observable length 
is a common feature of all promising quantum gravity candidates. The existence of a minimal measurable length modifies the Heisenberg uncertainty principle (HUP) to the so-called generalized (gravitational) uncertainty principle (GUP). On the other hand, Doubly Special Relativity Theories (for review see, for instance, [19-24]), suggest that existence of a minimal measurable length would restrict a test particle's momentum to take any arbitrary values. In other words, a test particle's momentum measurement cannot be arbitrarily imprecise leading nontrivially to an upper bound $P_{\max }$ on test particles' momentum. This means that there is a maximal particles' momentum because of fundamental structure of spacetime at the Planck scale [25-28]. Quantum field theory in the presence of minimal length as a natural ultraviolet cutoff has been studied by some authors [29-35]. Specially, Matsuo and Shibusa have studied quantization of fields based on a generalized uncertainty principle that admits just a minimal measurable length [34]. Also Shibusa has extended this study to the supersymmetric field theory based on the GUP with minimal length [35]. Since position and momentum are dual to each other, it is natural to argue that existence of a minimal measurable length naturally leads to the existence of a maximal momentum. This issue has not been considered in the mentioned studies of scalar field theory. It is obvious that existence of a cutoff on particles' momentum affects considerably the formulation of the quantum field theory with just a minimal length cutoff. Based on this argument, our central task in this study is to construct a field theory for free scalar fields in the presence of quantum gravity effects encoded in a GUP that admits existence of a minimal measurable length and a maximal particles' momentum. Following our recent work on Hilbert space representation of quantum mechanics in this case [36], we reformulate the main structure of a free scalar field theory in this setup. Our primary input is the following GUP [37-39]:

$$
[\widehat{x}, \hat{p}]=i \hbar\left(1-\beta p+2 \beta^{2} p^{2}\right)
$$

We investigate the quantization of free scalar field based on the deformed algebra (1.1) in the canonical formalism in $(1+1)$-dimensions and in the path integral formalism as well. In the process of quantization using the latter formalism, we use the Bjorken-Johnson-Low prescription $[40,41]$ which states that $T$-product of fields must take the same value in the two formalisms. We compare our results with Matsuo and Shibusa work [34] in each step.

\section{A Brief about GUP}

\subsection{GUP with a Minimal Length}

In ordinary quantum mechanics, the standard Heisenberg uncertainty principle (HUP) is given by

$$
\Delta x \Delta p \geq \frac{\hbar}{2}
$$

There is no trace of gravity in this relation. Today we know that HUP breaks down for energies close to the Planck scale where the corresponding Schwarzschild radius becomes comparable with the Compton wavelength with both becoming approximately of the order of the Planck length. As we have said in the Introduction, existence of a minimal measurable 
distance in quantum gravity is inevitable. This is encoded in the following Generalized (Gravitational) uncertainty principle (GUP)

$$
\Delta x \Delta p \geq \frac{\hbar}{2}+\frac{\beta_{0} l_{\mathrm{pl}}^{2}(\Delta p)^{2}}{\hbar} .
$$

The additional term, $\beta_{0} l_{\mathrm{pl}}^{2}(\Delta p)^{2} / \hbar$, has its origin on the very nature of space time at the Planck energy scale (see also [42-44] for alternative generalizations). It was shown in [17] that the simplest GUP relation which implies the appearance of a nonzero minimal uncertainty $\Delta x_{0}$ in position has the form

$$
\Delta x \Delta p \geq \frac{\hbar}{2\left(1+\beta(\Delta p)^{2}+\beta\langle p\rangle^{2}\right)},
$$

where $\beta$ is the GUP parameter defined as $\beta=\beta_{0} /\left(M_{\mathrm{pl}} c\right)^{2}=\beta_{0} l_{\mathrm{pl}}^{2} / \hbar^{2}$, and $M_{\mathrm{pl}} c^{2} \approx 10^{19} \mathrm{GeV}$ is the 4-dimensional fundamental scale.

At energies much below the Planck energy, the extra term in the right hand side of (2.2) would be irrelevant, which means $\beta \rightarrow 0$ and the standard HUP relation is recovered. Instead, approaching the Planck energy scale, this term becomes relevant and, as has been said, it is related to the minimal measurable length. From a string theory viewpoint since a string cannot probe distances smaller than its length, existence of a minimal observable length is reliable. Since for any pair of observable $A$ and $B$ (which are represented as symmetric operators on a domain of $\mathbf{A}^{2}$ and $\mathbf{B}^{2}$ ) one has [17]

$$
\Delta \mathbf{A} \Delta \mathbf{B} \geq \frac{\hbar}{2}|\langle[\mathbf{A}, \mathbf{B}]\rangle|,
$$

one find the following algebraic structure

$$
[x, p]=i \hbar\left(1+\beta p^{2}\right) \text {. }
$$

Following [17], we define position and momentum operators for the GUP case as

$$
\begin{gathered}
X=x, \\
P=p\left(1+\beta p^{2}\right),
\end{gathered}
$$

where $x$ and $p$ ensure the Jacobi identities, namely,

$$
\left[x_{i}, p_{j}\right]=i \hbar \delta_{i j}, \quad\left[x_{i}, x_{j}\right]=0, \quad\left[p_{i}, p_{j}\right]=0 .
$$

Now it is easy to show that $X$ and $P$ satisfy the generalized uncertainty principle. We interpret $p$ as the momentum operator at low energies which has the standard representation 
in position space, that is, $p_{j}=(\hbar / i)\left(\partial / \partial x_{j}\right)$, and $P$ as the momentum operator at high energies, which has the generalized representation in position space, that is, $P_{j}=$ $(\hbar / i)\left(\partial / \partial x_{j}\right)\left[1+\beta\left((\hbar / i)\left(\partial / \partial x_{j}\right)\right)^{2}\right]$.

\subsection{GUP with Minimal Length and Maximal Momentum}

Magueijo and Smolin have shown that in the context of the Doubly Special Relativity a test particle's momentum cannot be arbitrarily imprecise and therefore there is an upper bound for momentum fluctuation [25-27]. Then it has been shown by Cortes and Gamboa [28] that this may lead to a maximal measurable momentum for a test particle. In this framework, the GUP that predicts both a minimal observable length and a maximal momentum can be written as follows [37-39] (we note that maximal momentum arises also by considering the fact that the modification of the standard dispersion relation is such that the value of momentum saturates as one varies energy. See [45] for more details.):

$$
\Delta x \Delta p \geq \frac{\hbar}{2}\left(1-2 \beta\langle p\rangle+4 \beta^{2}\left\langle p^{2}\right\rangle\right)
$$

or

$$
\Delta x \Delta p \geq \frac{\hbar}{2}\left[1-\beta(\Delta p)+2 \beta^{2}(\Delta p)^{2}\right]
$$

In this framework the following algebraic structure can be deduced (see [37-39]):

$$
[x, p]=i \hbar\left(1-\beta p+2 \beta^{2} p^{2}\right),
$$

where $\beta$ is the GUP parameter in the presence of both minimal length and maximal momentum. Similar to the minimal length case, we can define [36]

$$
\begin{gathered}
X=x, \\
P=p\left(1-\beta p+2 \beta^{2} p^{2}\right),
\end{gathered}
$$

where as before $x$ and $p$ satisfy the canonical commutation relations via the Jacobi identity, and $X$ and $P$ satisfy the generalized commutation relations in the presence of minimal length and maximal momentum as

$$
[X, P]=i \hbar\left(1-\beta p+2 \beta^{2} p^{2}\right)
$$

\section{Variational Matrix Product State Approach}

We start with the following deformed Heisenberg algebra:

$$
[\widehat{x}, \hat{p}]=i \hbar\left(1-\beta p+2 \beta^{2} p^{2}\right)
$$


which has a representation in momentum space as

$$
\begin{gathered}
\hat{p}=p, \\
\widehat{x}=i \hbar\left(1-\beta p+2 \beta^{2} p^{2}\right) \frac{\partial}{\partial p} .
\end{gathered}
$$

In general, states which have minimal uncertainty obey the following equation:

$$
\left(\widehat{x}-\langle\widehat{x}\rangle+\frac{\langle[\Delta \widehat{x}, \Delta \hat{p}]\rangle}{2(\Delta \widehat{p})^{2}} \Delta \widehat{p}\right)|\psi\rangle=0
$$

where $\Delta \widehat{A} \equiv \widehat{A}-\langle\widehat{A}\rangle$ and $\Delta \widehat{A} \equiv \sqrt{\left\langle(\Delta \widehat{A})^{2}\right\rangle}$. Among these states, we will focus on the states which are subjected to the following conditions:

$$
\begin{aligned}
& (\Delta x)_{\min }=\hbar \sqrt{\beta}, \\
& (\Delta p)_{\min }=\frac{1}{\sqrt{\beta}},
\end{aligned}
$$

and for simplicity we set

$$
\langle\widehat{p}\rangle=0
$$

One can easily find the wavefunction of maximal localization states in momentum space as [36]

$$
\psi_{\zeta}(p)=\frac{N_{1}}{\sqrt{2 \pi \hbar}} \frac{1}{\sqrt{1-\beta p+2 \beta^{2} p^{2}}} \exp \left(\frac{\zeta}{i \hbar \sqrt{\beta}} \tan ^{-1}(\sqrt{\beta} p)\right)
$$

Here $\zeta \equiv\langle\widehat{x}\rangle$ and $N_{1}$ is a normalization factor. These maximal localization states are identified as one particle state in the theory based on GUP [36]. We will use these states as the basic ingredients in constructing quantum fields in forthcoming sections of this paper. Note that these states are not eigenstates of the position operator $\hat{x}$ because of limitation on resolution of space points encoded in the existence of minimal length. Nevertheless, momentum space representation is still applicable. So, we introduce a first quantized Heisenberg space as the space equipped with the complete basis $\{|p\rangle\}$ which has the completeness relation as

$$
\mathbf{1}=\int D_{p}|p\rangle\langle p|
$$


We determine the measure $D_{p}$ so that the operator $\widehat{x}=i \hbar\left(1-\beta p+2 \beta^{2} p^{2}\right)(\partial / \partial p)$ is Hermitian (i.e., $\langle\chi \mid \widehat{x} \psi\rangle=\langle\widehat{x} \chi \mid \psi\rangle$ )

$$
D_{p} \equiv N_{2} \frac{d p}{1-\beta p+2 \beta^{2} p^{2}}
$$

where $N_{2}$ is a normalization factor independent of $p$. We set $N_{2}=1$ by recalling the basis as $\sqrt{N_{2}}|p\rangle \rightarrow|p\rangle$. We note that this measure is not singular since the denominator of equation (3.8) has no real root. This measure leads to the orthogonality condition as

$$
\left\langle p \mid p^{\prime}\right\rangle=\left(1-\beta p+2 \beta^{2} p^{2}\right) \delta\left(p-p^{\prime}\right)
$$

We denote by $\left|\psi_{\zeta}\right\rangle$ the maximal localization states and then we can rewrite the wave function of one particle state (3.6) in the following form:

$$
\psi_{\zeta}(p)=\left\langle p \mid \psi_{\zeta}\right\rangle
$$

So we find

$$
i \hbar\left(1-\beta p+2 \beta^{2} p^{2}\right) \frac{\partial \psi_{\zeta}(p)}{\partial p}=\zeta \psi_{\zeta}(p)
$$

or

$$
\frac{\zeta}{i \hbar\left(1-\beta p+2 \beta^{2} p^{2}\right)} \partial p=\frac{\partial \psi_{\zeta}(p)}{\psi_{\zeta}(p)}
$$

Integrating this equation gives

$$
\ln \psi_{\zeta}(p)=\frac{\zeta}{i \hbar} \int_{-p_{\mathrm{pl}}}^{+p_{\mathrm{pl}}} \frac{d p}{\left(1-\beta p+2 \beta^{2} p^{2}\right)}=\frac{8 \zeta}{7 i \hbar} \int_{-p_{\mathrm{pl}}}^{+p_{\mathrm{pl}}} \frac{d p}{((4 \beta p-1) / \sqrt{7})^{2}+1}
$$

where the limits of integral are coming from existence of maximal momentum which is assumed to be the Planck momentum, $p_{\mathrm{pl}}$. So we find

$$
\psi_{\zeta}(p)=\psi_{\zeta}(0) \exp \left[\frac{-i 2 \zeta}{\beta \hbar \sqrt{7}}\left\{\tan ^{-1}\left(\frac{1}{\sqrt{7}}\right)+\tan ^{-1}\left(\frac{4 \beta p-1}{\sqrt{7}}\right)\right\}\right]
$$


Then, by normalization $\langle\psi \mid \psi\rangle=1$ we have

$$
\begin{aligned}
1 & =\int_{-p_{\mathrm{p} 1}}^{+p_{\mathrm{pl}}} \frac{d p}{\left(1-\beta p+2 \beta^{2} p^{2}\right)} \psi_{\zeta}^{*}(p) \psi_{\zeta}(p) \\
& =\psi_{\zeta}^{*}(0) \psi_{\zeta}(0) \int_{-p_{\mathrm{p} 1}}^{+p_{\mathrm{p} 1}} \frac{d p}{\left(1-\beta p+2 \beta^{2} p^{2}\right)} .
\end{aligned}
$$

So we have

$$
\psi_{\zeta}(0)=\sqrt{\frac{\beta \sqrt{7}}{2}}\left[\tan ^{-1}\left(\frac{4 \beta p_{\mathrm{pl}}-1}{\sqrt{7}}\right)+\tan ^{-1}\left(\frac{4 \beta p_{\mathrm{pl}}+1}{\sqrt{7}}\right)\right]^{-1 / 2} .
$$

Thus the formal position eigenvectors in momentum space are given by

$$
\begin{aligned}
\psi_{\zeta}(p)= & \sqrt{\frac{\beta \sqrt{7}}{2}}\left[\tan ^{-1}\left(\frac{4 \beta p_{\mathrm{pl}}-1}{\sqrt{7}}\right)+\tan ^{-1}\left(\frac{4 \beta p_{\mathrm{pl}}+1}{\sqrt{7}}\right)\right]^{-1 / 2} \\
& \times \exp \left[\frac{-i 2 \zeta}{\beta \hbar \sqrt{7}}\left\{\tan ^{-1}\left(\frac{1}{\sqrt{7}}\right)+\tan ^{-1}\left(\frac{4 \beta p-1}{\sqrt{7}}\right)\right\}\right] .
\end{aligned}
$$

This is the generalized, momentum space eigenstate of the position operator in the presence of both a minimal length and a maximal momentum. Now we calculate the scaler product of the formal position eigenstate as follows:

$$
\begin{aligned}
\left\langle\psi_{\zeta^{\prime}} \mid \psi_{\zeta}\right\rangle= & \int_{-p_{\mathrm{pl}}}^{+p_{\mathrm{pl}}} \frac{d p}{\left(1-\beta p+2 \beta^{2} p^{2}\right)} \psi_{\zeta^{\prime}}^{*}(p) \psi_{\zeta}(p) \\
= & \frac{\beta \sqrt{7}}{2}\left[\tan ^{-1}\left(\frac{4 \beta p_{\mathrm{pl}}-1}{\sqrt{7}}\right)+\tan ^{-1}\left(\frac{4 \beta p_{\mathrm{pl}}+1}{\sqrt{7}}\right)\right]^{-1} \int_{-p_{\mathrm{pl}}}^{+p_{\mathrm{pl}}} \frac{d p}{\left(1-\beta p+2 \beta^{2} p^{2}\right)} \\
& \quad \exp \left(\frac{2\left(\zeta-\zeta^{\prime}\right)}{i \hbar \beta \sqrt{7}}\left\{\tan ^{-1}\left(\frac{1}{\sqrt{7}}\right)+\tan ^{-1}\left(\frac{4 \beta p-1}{\sqrt{7}}\right)\right\}\right) \\
= & i \lambda\left[\exp \left(\frac{2\left(\zeta-\zeta^{\prime}\right)}{i \hbar \beta \sqrt{7}}\left\{\tan ^{-1}\left(\frac{1}{\sqrt{7}}\right)+\tan ^{-1}\left(\frac{4 \beta p_{\mathrm{pl}}-1}{\sqrt{7}}\right)\right\}\right)\right. \\
& \left.\quad-\exp \left(\frac{2\left(\zeta-\zeta^{\prime}\right)}{i \hbar \beta \sqrt{7}}\left\{\tan ^{-1}\left(\frac{1}{\sqrt{7}}\right)-\tan ^{-1}\left(\frac{4 \beta p_{\mathrm{pl}}+1}{\sqrt{7}}\right)\right\}\right)\right]
\end{aligned}
$$


where by definition

$$
\lambda \equiv \frac{\hbar \beta \sqrt{7}}{2\left(\zeta-\zeta^{\prime}\right)}\left[\tan ^{-1}\left(\frac{4 \beta p_{\mathrm{pl}}-1}{\sqrt{7}}\right)+\tan ^{-1}\left(\frac{4 \beta p_{\mathrm{pl}}+1}{\sqrt{7}}\right)\right]^{-1}
$$

Furthermore, we find a completeness of basis $\left\{\left|\psi_{\zeta}\right\rangle\right\}$ on this Hilbert space as

$$
\begin{aligned}
1 & =\int_{-p_{\mathrm{p} 1}}^{+p_{\mathrm{p} 1}} \frac{d \zeta}{\left|N_{1}\right|^{2}}\left(1-\beta p+2 \beta^{2} p^{2}\right)\left|\psi_{\zeta}\right\rangle\left\langle\psi_{\zeta}\right| \\
& =\int_{-p_{\mathrm{p} 1}}^{+p_{\mathrm{p} 1}} \frac{d \zeta}{\left|N_{1}\right|^{2}}\left|\psi_{\zeta}\right\rangle\left\langle\psi_{\zeta}\right|\left(1-\beta p+2 \beta^{2} p^{2}\right) .
\end{aligned}
$$

In what follows we set $N_{1}=1$ by rescaling the basis as $\left(N_{1}\right)^{-1}\left|\psi_{\zeta}\right\rangle \rightarrow\left|\psi_{\zeta}\right\rangle$. Here we have two bases $\{|p\rangle\}$ and $\left\{\left|\psi_{\zeta}\right\rangle\right\}$. Thus we can perform a Fourier transformation which interchanges the two representations. We write the $p$-space wavefunction and $\zeta$-space wavefunction for a state $|\phi\rangle$ in this Hilbert space as follows (See [36] for more details):

$$
\begin{aligned}
& \phi(p) \equiv\langle p \mid \phi\rangle, \\
& \phi(\zeta) \equiv\left\langle\psi_{\zeta} \mid \phi\right\rangle .
\end{aligned}
$$

The Fourier transformation in this Hilbert space is

$$
\begin{aligned}
\phi(\zeta) & =\int_{-p_{\mathrm{pl}}}^{+p_{\mathrm{pl}}} \frac{d p}{\left(1-\beta p+2 \beta^{2} p^{2}\right)} \psi_{\zeta}^{*}(p) \phi(p) \\
& =\int_{-p_{\mathrm{p} 1}}^{+p_{\mathrm{pl}}} \frac{\left|N_{1}\right|}{\sqrt{2 \pi \hbar}} \frac{d p}{\left(1-\beta p+2 \beta^{2} p^{2}\right)^{3 / 2}} \phi(p) \exp \left(\frac{i \zeta}{\hbar \sqrt{\beta}} \tan ^{-1}(\sqrt{\beta p})\right), \\
\phi(p)= & \int_{-p_{\mathrm{p} 1}}^{+p_{\mathrm{pl}}} d \zeta\left(1-\beta p+2 \beta^{2} p^{2}\right) \psi_{\zeta}(p) \phi(\zeta) \\
= & \int_{-p_{\mathrm{p} 1}}^{+p_{\mathrm{pl}}} \frac{\left(1-\beta p+2 \beta^{2} p^{2}\right)^{1 / 2}}{\sqrt{2 \pi \hbar}} \exp \left(\frac{-i \zeta}{\hbar \sqrt{\beta}} \tan ^{-1}(\sqrt{\beta} p)\right) \phi(\zeta) d \zeta .
\end{aligned}
$$

Here there are some points which are crucial to be mentioned. The transformation (3.23) is an inverse transformation to (3.22) for arbitrary square integrable functions as in the case of usual Fourier transformation. However the transformation (3.22) is not an inverse 
transformation to (3.23) for arbitrary square integrable functions. This can be seen easily by inserting (3.23) into (3.22), then we find

$$
\begin{aligned}
& \int_{-p_{\mathrm{pl}}}^{+p_{\mathrm{pl}}} \frac{d p}{\left(1-\beta p+2 \beta^{2} p^{2}\right)} \psi_{\zeta}^{*}(p) \phi(p) \\
& =\int_{-p_{\mathrm{pl}}}^{+p_{\mathrm{pl}}} \frac{d p}{\left(1-\beta p+2 \beta^{2} p^{2}\right)} \psi_{\zeta}^{*}(p) \int_{-p_{\mathrm{pl}}}^{+p_{\mathrm{pl}}}\left(1-\beta p+2 \beta^{2} p^{2}\right) \psi_{\zeta^{\prime}}(p) \phi\left(\zeta^{\prime}\right) d \zeta^{\prime} \\
& =\int_{-p_{\mathrm{pl}}}^{+p_{\mathrm{pl}}} \frac{d p}{\left(1-\beta p+2 \beta^{2} p^{2}\right)} \psi_{\zeta}^{*}(p) \psi_{\zeta^{\prime}}(p) \int_{-p_{\mathrm{pl}}}^{+p_{\mathrm{pl}}}\left(1-\beta p+2 \beta^{2} p^{2}\right) \phi\left(\zeta^{\prime}\right) d \zeta^{\prime} \\
& =\int_{-p_{\mathrm{pl}}}^{+p_{\mathrm{pl}}} d p \frac{\beta \sqrt{7}}{2}\left[\tan ^{-1}\left(\frac{4 \beta p_{\mathrm{pl}}-1}{\sqrt{7}}\right)+\tan ^{-1}\left(\frac{4 \beta p_{\mathrm{pl}}+1}{\sqrt{7}}\right)\right]^{-1} \\
& \times \exp \left(\frac{2\left(\zeta-\zeta^{\prime}\right)}{i \hbar \beta \sqrt{7}}\left\{\tan ^{-1}\left(\frac{1}{\sqrt{7}}\right)+\tan ^{-1}\left(\frac{4 \beta p-1}{\sqrt{7}}\right)\right\}\right) \phi\left(\zeta^{\prime}\right) d \zeta^{\prime} \\
& =\int_{-p_{\mathrm{pl}}}^{+p_{\mathrm{pl}}} i \lambda\left[e^{N(A+C)}-e^{(-N)(B-C)}\right] d \zeta^{\prime}\left(1-\beta p+2 \beta^{2} p^{2}\right) \phi\left(\zeta^{\prime}\right),
\end{aligned}
$$

where by definition

$$
\begin{gathered}
\lambda \equiv \frac{\hbar \beta \sqrt{7}}{2\left(\zeta-\zeta^{\prime}\right)}\left[\tan ^{-1}\left(\frac{4 \beta p_{\mathrm{pl}}-1}{\sqrt{7}}\right)+\tan ^{-1}\left(\frac{4 \beta p_{\mathrm{pl}}+1}{\sqrt{7}}\right)\right]^{-1}, \\
A \equiv \tan ^{-1}\left(\frac{4 \beta p_{\mathrm{pl}}-1}{\sqrt{7}}\right) \\
B \equiv \tan ^{-1}\left(\frac{4 \beta p_{\mathrm{pl}}+1}{\sqrt{7}}\right) \\
C \equiv \tan ^{-1}\left(\frac{1}{7}\right) \\
N \equiv \frac{2\left(\zeta-\zeta^{\prime}\right)}{i \hbar \beta \sqrt{7}}
\end{gathered}
$$

This is not equal to the original function for arbitrary function $\phi\left(\zeta^{\prime}\right)$. We restrict the function $\phi\left(\zeta^{\prime}\right)$ to liner combination of $\psi_{\zeta^{\prime}}^{*}(p)^{\prime} s$ as

$$
\phi\left(\zeta^{\prime}\right)=\int_{-p_{\mathrm{pl}}}^{+p_{\mathrm{pl}}} \frac{d p}{\left(1-\beta p+2 \beta^{2} p^{2}\right)} a(p) \psi_{\zeta^{\prime}}^{*}(p)
$$


For these functions, the transformation (3.22) is as follows:

$$
\phi\left(\zeta^{\prime}\right)=\int_{-p_{\mathrm{pl}}}^{+p_{\mathrm{pl}}} \frac{d p}{\left(1-\beta p+2 \beta^{2} p^{2}\right)} a(p) \psi_{\zeta^{\prime}}^{*}(p) .
$$

Now for these functions the transformation (3.22) is truly the inverse transformation to (3.23). That is,

$$
\begin{aligned}
\int_{-p_{\mathrm{pl}}}^{+p_{\mathrm{pl}}} \frac{d p}{\left(1-\beta p+2 \beta^{2} p^{2}\right)} \psi_{\zeta}^{*}(p) \phi(p) & =\int_{-p_{\mathrm{pl}}}^{+p_{\mathrm{pl}}} d \zeta^{\prime}\left(1-\beta p+2 \beta^{2} p^{2}\right) \psi_{\zeta^{\prime}}(p) \phi\left(\zeta^{\prime}\right) \\
& =\int_{-p_{\mathrm{pl}}}^{+p_{\mathrm{p} 1}} \frac{d p d p^{\prime} d \zeta^{\prime}}{\left(1-\beta p+2 \beta^{2} p^{2}\right)} a\left(p^{\prime}\right) \psi_{\zeta}^{*}(p) \psi_{\zeta^{\prime}}(p) \psi_{\zeta^{\prime}}^{*}\left(p^{\prime}\right) \\
& =\int_{-p_{\mathrm{pl}}}^{+p_{\mathrm{pl}}} \frac{d p^{\prime}}{\left(1-\beta p+2 \beta^{2} p^{2}\right)} a\left(p^{\prime}\right) \psi_{\zeta}^{*}\left(p^{\prime}\right)=\phi(\zeta) .
\end{aligned}
$$

Since

$$
\begin{gathered}
\int_{-p_{\mathrm{pl}}}^{+p_{\mathrm{pl}}} d p d \zeta^{\prime} \psi_{\zeta}^{*}(p) \psi_{\zeta^{\prime}}(p)=\int_{-p_{\mathrm{pl}}}^{+p_{\mathrm{pl}}} \frac{\left(1-\beta p^{\prime}+2 \beta^{2} p^{\prime 2}\right)}{\left(1-\beta p^{\prime}+2 \beta^{2} p^{\prime 2}\right)} d p \psi_{\zeta}^{*}(p) \psi_{\zeta^{\prime}}^{\prime}(p) d \zeta^{\prime}, \\
\int_{-p_{\mathrm{pl}}}^{+p_{\mathrm{p} 1}}\left(1-\beta p^{\prime}+2 \beta^{2} p^{\prime 2}\right)\left\langle\psi_{\zeta} \mid \psi_{\zeta^{\prime}}\right\rangle=\int_{-p_{\mathrm{pl}}}^{+p_{\mathrm{pl}}}\left(1-\beta p^{\prime}+2 \beta^{2} p^{\prime 2}\right) \delta\left(\zeta-\zeta^{\prime}\right) d \zeta^{\prime}=1 .
\end{gathered}
$$

This restriction is nothing but the condition that the state $|\phi\rangle$ is included in our Hilbert space. Thus the Fourier transformation, (3.22) and (3.23), is well defined in this Hilbert space. It should be noted that the wave function (3.6) reduces to the plane wave solution in the limit of $\beta \rightarrow 0$

$$
\psi_{\zeta}(p) \longrightarrow \frac{1}{\sqrt{2 \pi \hbar}} \exp \left(\frac{\zeta p}{i \hbar}\right)
$$

This is because we have set the normalization constant as $N_{1}=1$ by rescaling $\left|\psi_{\zeta}\right\rangle$. In [36] the normalization constant is fixed to $N_{1}=2 \sqrt{\hbar \sqrt{\beta}}$ so that the norm of the wavefunction is equal to one. In this case, the wavefunction vanishes in the mentioned limit because of the extra dependence on $\beta$ in the normalization factor.

\section{A (1+1)-Dimensional Quantum Field Theory with GUP}

In this section we construct a quantum field theory with a GUP (that admits both a minimal length and a maximal momentum) in $(1+1)$-dimensional spacetime in the canonical 
formalism. First of all, we define a classical field such that it solves the following KleinGordon equation (see e.g., [46]):

$$
\begin{gathered}
-\hbar^{2} \frac{\partial^{2} \Phi}{\partial t^{2}}=-\hbar^{2} c^{2} \nabla^{2} \Phi+m^{2} c^{4} \Phi, \\
0=\left[\left(\hbar \frac{\partial}{\partial t}\right)^{2}+Z(p)+m^{2}\right] \Phi(p, t) .
\end{gathered}
$$

In this equation we leave the function of momentum $Z(p)$ indefinite, where $Z(p)$ is an arbitrary even function whose explicit form does not have any influence on the following arguments [34]. This function determines the dispersion relation and depends on what kind of theory we want. Quantization of classical field is in order to provide the set of operator fields and Hamiltonian that lead the same Klein-Gordon equation by the Heisenberg equation. We define a field $\widehat{\Phi}(p, t)$ (see for instance [34])

$$
\widehat{\Phi}(p, t)=\frac{\hbar}{\sqrt{E(p)}} \widehat{\phi}(p) \exp \left(\frac{t}{i \hbar} E(p)\right)+\frac{\hbar}{\sqrt{E(-p)}} \widehat{\phi}^{\dagger}(-p) \exp \left(\frac{-t}{i \hbar} E(-p)\right)
$$

where $E(p) \equiv \sqrt{Z(p)+m^{2}}$. This field satisfies the Klein-Gordon equation (4.1). Its Fourier pair $\widehat{\phi}(\zeta, t)$ is written in terms of our one-particle states in space momentum

$$
\begin{aligned}
\widehat{\Phi}(\zeta, t) \equiv \int_{-p_{\mathrm{pl}}}^{+p_{\mathrm{p} 1}} \frac{d p}{\left(1-\beta p+2 \beta^{2} p^{2}\right)}\left\{\psi_{\zeta}^{*}(p) \widehat{\phi}(p) \frac{\hbar}{\sqrt{2 E(p)}} \exp \left(\frac{1}{i \hbar} t E(p)\right)\right. \\
\left.+\psi_{\zeta}(p) \hat{\phi}^{\dagger}(p) \frac{\hbar}{\sqrt{2 E(p)}} \exp \left(\frac{-1}{i \hbar} t E(p)\right)\right\} .
\end{aligned}
$$

In the above expression, the operators $\widehat{\phi}(p)$ and $\widehat{\phi}^{\dagger}(p)$ are, respectively, the annihilation and creation operators for one particle state with momentum $p$. They have the following commutation relation in momentum space:

$$
\left[\widehat{\phi}(p), \hat{\phi}^{\dagger}\left(p^{\prime}\right)\right]=\left(1-\beta p+2 \beta^{2} \mathrm{p}^{2}\right) \delta\left(p-p^{\prime}\right)
$$

We construct a natural Hamiltonian in terms of the Fock space picture

$$
\mathscr{H} \equiv \int_{-p_{\mathrm{pl}}}^{+p_{\mathrm{pl}}} \frac{d p}{\left(1-\beta p+2 \beta^{2} p^{2}\right)} E(p) \widehat{\phi}^{\dagger}(p) \widehat{\phi}(p)
$$


This Hamiltonian produces the following Heisenberg equation of motion:

$$
\frac{1}{i \hbar}[\widehat{\Phi}(p, t), \mathscr{l}]=\frac{\partial}{\partial t} \widehat{\Phi}(p, t)
$$

which is consistent with the Klein-Gordon equation (4.1). We can define the conjugate momentum $\widehat{\Pi}(p, t) \equiv(\partial / \partial t) \widehat{\Phi}(p, t)$. Therefore, we can write the Hamiltonian in momentum space as

$$
\mathscr{l} \equiv \int_{-p_{\mathrm{pl}}}^{+p_{\mathrm{pl}}} \frac{d p}{\left(1-\beta p+2 \beta^{2} p^{2}\right)} \frac{1}{2}\left[\widehat{\Pi}(-p, t) \widehat{\Pi}(p, t)+\left(\frac{E(p)}{\hbar}\right)^{2} \widehat{\Phi}(-p, t) \times \widehat{\Phi}(p, t)\right]+\text { const.. }
$$

Now we compute the following commutation relation using the commutation relation (4.4):

$$
\left[\widehat{\Phi}(p, t), \widehat{\Pi}\left(p^{\prime}, t\right)\right]=i \hbar\left(1-\beta p+2 \beta^{2} p^{2}\right) \delta\left(p+p^{\prime}\right)
$$

We have also

$$
\begin{aligned}
{\left[\widehat{\Phi}(\zeta, t), \widehat{\Pi}\left(\zeta^{\prime}, t\right)\right]=} & \mathrm{i} \hbar\left\langle\psi_{\zeta} \mid \psi_{\zeta^{\prime}}\right\rangle=\frac{-\sqrt{7} \beta \hbar^{2}}{2\left(\zeta-\zeta^{\prime}\right)}\left[\tan ^{-1}\left(\frac{4 \beta p_{\mathrm{pl}}-1}{\sqrt{7}}\right)+\tan ^{-1}\left(\frac{4 \beta p_{\mathrm{pl}}+1}{\sqrt{7}}\right)\right]^{-1} \\
\times & \left\{\exp \left[\frac{2\left(\zeta-\zeta^{\prime}\right)}{i \hbar \beta \sqrt{7}}\left(\tan ^{-1}\left(\frac{1}{\sqrt{7}}\right)+\tan ^{-1}\left(\frac{4 \beta p_{\mathrm{pl}}-1}{\sqrt{7}}\right)\right)\right]\right. \\
& \left.\quad-\exp \left[\frac{-2\left(\zeta-\zeta^{\prime}\right)}{i \hbar \beta \sqrt{7}}\left(\tan ^{-1}\left(\frac{1}{\sqrt{7}}\right)+\tan ^{-1}\left(\frac{4 \beta p_{\mathrm{pl}}+1}{\sqrt{7}}\right)\right)\right]\right\} .
\end{aligned}
$$

\section{Higher Dimensional Algebra}

The generalization of the Heisenberg algebra to higher dimensions where rotation symmetry is preserved and there are both a minimal length and a maximal momentum is (see [36])

$$
\left[\widehat{x}_{i}, \widehat{p}_{j}\right]=i \hbar \delta_{i j}\left(1-\beta p+2 \beta^{2} p^{2}\right)
$$

where in three dimensions $\vec{p}=p_{x} \vec{i}+p_{y} \vec{j}+p_{z} \vec{k}$, that is, $\vec{i}, \vec{j}$, and $\vec{k}$ are unit vectors of cartesian coordinates and $(\vec{p})^{2}=\sum_{i=1}^{d}\left(\widehat{p}_{i}\right)^{2}$. So, we can generalize our operators acting on momentum space in higher dimensional theory as

$$
\begin{gathered}
\mathbf{p}_{i} \varphi(p)=p_{i} \varphi(p) \\
\mathbf{X}_{\mathbf{j}} \varphi(p)=i \hbar\left(1-\beta \mathbf{p}+2 \beta^{2} \mathbf{p}^{2}\right) \frac{\partial}{\partial p_{j}} \varphi(p)
\end{gathered}
$$


Then, it is easy to show that

$$
\left[\mathbf{X}_{i}, \mathbf{X}_{j}\right]=i \beta \hbar\left(\frac{4 \beta P-1}{P}\right)\left(\mathbf{P}_{i} \mathbf{X}_{j}-\mathbf{P}_{j} \mathbf{X}_{i}\right)
$$

We see that in the presence of minimal length and maximal momentum, the very spacetime manifold is noncommutative in nature. Note that it was not the case for Matsuo and Shibusa analysis in the presence of just the minimal length [34]. So incorporation of natural cutoffs as minimal length and maximal momentum brings the spacetime manifold to be noncommutative in quantum gravity era. The generator of rotation in our framework are [36]

$$
\begin{gathered}
\mathbf{L}_{i j}=\frac{1}{\left(1-\beta \mathbf{p}+2 \beta^{2} \mathbf{p}^{2}\right)}\left(\mathbf{X}_{i} \mathbf{P}_{j}-\mathbf{X}_{j} \mathbf{P}_{i}\right) \\
{\left[\mathbf{X}_{i}, \mathbf{X}_{j}\right]=-i \beta \hbar\left(4 \beta-\frac{1}{\mathbf{p}}\right)\left(1-\beta \mathbf{p}+2 \beta^{2} \mathbf{p}^{2}\right) \mathbf{L}_{i j}}
\end{gathered}
$$

As Kempf et al. [17] case, from the positivity of the norm, that is,

$$
\|\left(X-\langle X\rangle+\frac{\langle[X, P]\rangle}{2(\Delta P)^{2}}(P-\langle P\rangle)\right)|\varphi\rangle \| \geq 0,
$$

on the boundary of the physically allowed region, we have

$$
\left(X-\langle X\rangle+\frac{\langle[X, P]\rangle}{2(\Delta P)^{2}}(P-\langle P\rangle)\right)|\varphi\rangle=0
$$

Using (5.2), the differential equation in momentum space corresponding to (5.6) is in the following form:

$$
\left[i \hbar\left(1-\beta p+2 \beta^{2} p^{2}\right) \frac{\partial}{\partial p_{i}}-\left\langle\widehat{X}^{i}\right\rangle+i \hbar \times \frac{1+2 \beta^{2}(\Delta p)^{2}+2 \beta^{2}\langle p\rangle^{2}-\beta\langle p\rangle}{2(\Delta p)^{2}}\left(p_{i}-\left\langle p_{i}\right\rangle\right)\right]|\varphi\rangle=0 .
$$

By introducing the path integral measure and also Lagrangian through introduction of two parameters $\ell$ and $m$ defined as follows:

$$
\begin{aligned}
& 1=\int_{-p_{\mathrm{pl}}}^{+p_{\mathrm{pl}}} D \Phi(p, t) \exp \left(-\frac{1}{2} \int_{-p_{\mathrm{pl}}}^{+p_{\mathrm{pl}}} \frac{d t d p}{\left(1-\beta p+2 \beta^{2} p^{2}\right)^{\ell}} \Phi(p, t) \Phi(-p, t)\right) \\
& \mathcal{L}=-\frac{1}{2} \int_{-p_{\mathrm{p} 1}}^{+p_{\mathrm{p} 1}} d p\left(1-\beta p+2 \beta^{2} p^{2}\right)^{m} \Phi(-p, t)\left[\partial_{t}^{2}+\left(\frac{E(p)}{\hbar}\right)^{2}\right] \Phi(p, t),
\end{aligned}
$$


the action $S \equiv \int d t \mathcal{L}$ of the model can be written as follows:

$$
\begin{aligned}
S= & -\frac{1}{2} \int_{-p_{p l}}^{+p_{p l}} d p d p^{\prime} d q d q^{\prime}\left(1-\beta p+2 \beta^{2} p^{2}\right)^{m} \delta\left(p+p^{\prime}\right) \delta\left(q+q^{\prime}\right) \\
& \times \Phi(p, q)\left[-\left(\frac{q}{\hbar}\right)^{2}+\left(\frac{E(p)}{\hbar}\right)^{2}\right] \Phi\left(p^{\prime}, q^{\prime}\right) .
\end{aligned}
$$

Now using assumptions (5.8) and (5.10), we can compute the $T^{*}$-product in this framework as follows:

$$
\left\langle T^{*} \widehat{\Phi}(p, q) \widehat{\Phi}\left(p^{\prime}, q^{\prime}\right)\right\rangle=\frac{\hbar^{3}}{i}\left(1-\beta p+2 \beta^{2} p^{2}\right)^{-m-\ell} \delta\left(p+p^{\prime}\right) \delta\left(q+q^{\prime}\right) \times \frac{1}{-q^{2}+E^{2}(p)-i \varepsilon} .
$$

Using the Bjorken-Johnson-Low prescription, from behavior of

$$
\begin{aligned}
& \lim _{q \rightarrow \infty}\left\langle T^{*} \widehat{\Phi}(p, q) \widehat{\Phi}\left(p^{\prime}, q^{\prime}\right)\right\rangle, \\
& \lim _{q \rightarrow \infty} q\left\langle T^{*} \widehat{\Phi}(p, q) \widehat{\Phi}\left(p^{\prime}, q^{\prime}\right)\right\rangle,
\end{aligned}
$$

we find

$$
T^{*} \widehat{\Phi}(p, q) \widehat{\Phi}\left(p^{\prime}, q^{\prime}\right)=T \widehat{\Phi}(p, q) \widehat{\Phi}\left(p^{\prime}, q^{\prime}\right), \quad\left[\widehat{\Phi}(p, t), \widehat{\Phi}\left(p^{\prime}, t\right)\right]=0
$$

Also through the behavior of $\lim _{q \rightarrow \infty} q^{2}\left\langle T^{*} \widehat{\Phi}(p, q) \widehat{\Phi}\left(p^{\prime}, q^{\prime}\right)\right\rangle$, we can obtain

$$
\left[\widehat{\Phi}(p, t), \dot{\hat{\Phi}}\left(p^{\prime}, t\right)\right]=i \hbar\left(1-\beta p+2 \beta^{2} p^{2}\right)^{-m-\ell} \delta\left(p+p^{\prime}\right)
$$

If we define conjugate momentum $\Pi(p, t)$ in Lagrangian Formalism by using a free parameter $r$ as

$$
\Pi(p, t) \equiv\left(1-\beta p+2 \beta^{2} p^{2}\right)^{r} \frac{\delta S}{\delta \Phi(-p, t)}
$$

the commutation relation (5.14) takes the following form:

$$
\left[\widehat{\Phi}(p, t), \widehat{\Pi}\left(p^{\prime}, t\right)\right]=i \hbar\left(1-\beta p+2 \beta^{2} p^{2}\right)^{-2 m-\ell-r} \delta\left(p+p^{\prime}\right) .
$$


The right hand side of the above equation is a symplectic form [34]. Since the Legender transformation from Lagrangian to Hamiltonian is (see [34])

$$
\frac{1}{i \hbar} \int \mathscr{\ell} d t \equiv(\text { symplectic form })^{-1} \sum_{i} \Pi_{i} d \Phi_{i}-\frac{1}{i \hbar} \int d t \mathcal{L}
$$

one can obtain a Hamiltonian as

$$
\begin{aligned}
\mathscr{H}=\int_{-p_{\mathrm{p} 1}}^{+p_{\mathrm{pl}}} d p\left(1-\beta p+2 \beta^{2} p^{2}\right)^{m+\ell}[ & \left\{1-\frac{1}{2}\left(1-\beta p+2 \beta^{2} p^{2}\right)^{-2 m-2 \ell-2 r}\right\} \widehat{\Pi}(-p, t) \widehat{\Pi}(p, t) \\
& \left.+\frac{1}{2}\left(1-\beta p+2 \beta^{2} p^{2}\right)^{-\ell}\left(\frac{E(p)}{\hbar}\right)^{2} \widehat{\Phi}(-p, t) \widehat{\Phi}(p, t)\right] .
\end{aligned}
$$

The condition that the coefficient of $\Pi(-p, t) \Pi(p, t)$ must be homogeneous fixes the free parameter $r$ so that

$$
-\ell-2 m-2 r=0
$$

So we find

$$
r=-\frac{\ell}{2}-m
$$

Then the Hamiltonian becomes

$$
\begin{aligned}
\mathscr{H}= & \int_{-p_{\mathrm{pl}}}^{+p_{\mathrm{pl}}} d p\left(1-\beta p+2 \beta^{2} p^{2}\right)^{m+\ell} \\
& \times \frac{1}{2}\left[\widehat{\Pi}(-p, t) \widehat{\Pi}(p, t)+\left(1-\beta p+2 \beta^{2} p^{2}\right)^{-\ell}\left(\frac{E(p)}{\hbar}\right)^{2} \widehat{\Phi}(-p, t) \widehat{\Phi}(p, t)\right] .
\end{aligned}
$$

By comparing this with (4.7), we obtain the parameters $m$ and $\ell$ as the form

$$
\begin{gathered}
\ell=0, \\
m=-1 .
\end{gathered}
$$


We see explicitly that the two formalisms provide consistent structure of the deformed quantum field theory. Summarizing the results, we have

$$
\begin{aligned}
\mathscr{H}= & \int_{-p_{\mathrm{pl}}}^{+p_{\mathrm{pl}}} d p\left(1-\beta p+2 \beta^{2} p^{2}\right)^{-1} \\
& \times \frac{1}{2}\left[\widehat{\Pi}(-p, t) \widehat{\Pi}(p, t)+\left(\frac{E(p)}{\hbar}\right)^{2} \widehat{\Phi}(-p, t) \widehat{\Phi}(p, t)\right]+\text { const. } \\
= & \int_{-p_{\mathrm{pl}}}^{+p_{\mathrm{pl}}} d p\left(1-\beta p+2 \beta^{2} p^{2}\right)^{-1} E(p)\left(\widehat{\phi}^{\dagger}(p) \widehat{\phi}(p)+\text { const. }\right) .
\end{aligned}
$$

Now the annihilation and creation operators for one particle states in the presence of both minimal length and maximal momentum have the following commutation relation

$$
\left[\widehat{\phi}(p), \hat{\phi}^{\dagger}\left(p^{\prime}\right)\right]=\left(1-\beta p+2 \beta^{2} p^{2}\right) \delta\left(p-p^{\prime}\right),
$$

and the canonical commutation relation is

$$
\left[\widehat{\Phi}(p, t), \widehat{\Pi}\left(p^{\prime}, t\right)\right]=i \hbar\left(1-\beta p+2 \beta^{2} p^{2}\right) \delta\left(p+p^{\prime}\right) .
$$

Also Heisenberg equation of motion is given as

$$
\frac{1}{i \hbar}[\widehat{\Phi}(p, t), \mathscr{d}]=\frac{\partial}{\partial t} \widehat{\Phi}(p, t)=\widehat{\Pi}(p, t) .
$$

Now we calculate propagators from these quantities in the presence of minimal length and maximal momentum as follows:

$$
\begin{aligned}
\left\langle T \widehat{\Phi}(p, q) \widehat{\Phi}\left(p^{\prime}, q^{\prime}\right)\right\rangle & \equiv \frac{1}{2 \pi \hbar} \int_{-p_{\mathrm{pl}}}^{+p_{\mathrm{pl}}} d t d t^{\prime} \exp \left(-\frac{q t+q^{\prime} t^{\prime}}{i \hbar}\right)\left\langle T \widehat{\Phi}(p, t) \widehat{\Phi}\left(p^{\prime}, t^{\prime}\right)\right\rangle \\
& =\frac{\hbar^{3}}{i}\left(1-\beta p+2 \beta^{2} p^{2}\right) \delta\left(p+p^{\prime}\right) \delta\left(q+q^{\prime}\right) \frac{1}{-q^{2}+E^{2}(p)-i \varepsilon} .
\end{aligned}
$$

In this case the Lagrangian is given by

$$
\mathcal{L}=-\frac{1}{2} \int_{-p_{\mathrm{pl}}}^{+p_{\mathrm{pl}}} d p\left(1-\beta p+2 \beta^{2} p^{2}\right)^{-1} \Phi(-p, t)\left[\partial_{t}^{2}+\left(\frac{E(p)}{\hbar}\right)^{2}\right] \Phi(p, t) .
$$

Also

$$
\mathbf{1}=\int_{-p_{\mathrm{p} 1}}^{+p_{\mathrm{pl}}} D \Phi(p, t) \exp \left(-\frac{1}{2} \int_{-p_{\mathrm{p} 1}}^{+p_{\mathrm{pl}}} d t d p \Phi(p, t) \Phi(-p, t)\right)
$$


Lagrangian and path integral measure in $(d+1)$-dimensional spacetime and in the presence of both minimal length and maximal momentum can be written a follows:

$$
\begin{gathered}
\mathcal{L}=-\frac{1}{2} \int_{-p_{\mathrm{p} 1}}^{+p_{\mathrm{p} 1}} d^{d} p\left(1-\beta \vec{p}+2 \beta^{2} \vec{p}^{2}\right)^{-1} \Phi(-\vec{p}, t)\left[\partial_{t}^{2}+\left(\frac{E(\vec{p})}{\hbar}\right)^{2}\right] \Phi(\vec{p}, t), \\
\mathbf{1}=\int_{-p_{\mathrm{p} 1}}^{+p_{\mathrm{p} 1}} D \Phi(\vec{p}, t) \exp \left(-\frac{1}{2} \int_{-p_{\mathrm{pl}}}^{+p_{\mathrm{p} 1}} d t d^{d} p \Phi(\vec{p}, t) \Phi(-\vec{p}, t)\right) .
\end{gathered}
$$

So we have provided the basic ingredients of a scalar field theory in the context of minimal length and maximal momentum hypothesis. We note that incorporation of maximal momentum as a new natural cutoff in quantum field theory restricts further the form of commutation relations and propagators.

\section{Summary}

Incorporation of quantum gravity effects in quantum mechanics and also quantum field theory leads to the existence of natural cutoffs on position and momentum measurements of test particles. These natural cutoffs regularize the underlying field theory in a phenomenologically viable manner. Quantum field theory of scalar fields in the presence of minimal length has been studied in literature (see [34]). Here, following our recent work on Hilbert space representation of quantum mechanics with minimal length and maximal momentum [36], we generalized the work reported in [34] to the case that there is a maximal momentum for test particles too. We have formulated a quantum theory of free scalar fields in $(1+1)$-dimensions based on a deformed Heisenberg algebra $[\widehat{x}, \hat{p}]=i \hbar\left(1-\beta p+2 \beta^{2} p^{2}\right)$, where $\beta$ is a deformation parameter. The generalized uncertainty principle based on this algebra requires the existence of a minimal measurable length and also maximal measurable momentum of the order of Planck momentum for any test particle. In this framework we have considered both canonical and path integral quantization of free scalar field in the presence of these natural cutoffs. Finally we have extended our analysis to a higher dimensional spacetime in the path integral formalism. We have shown that in the presence of minimal length and maximal momentum, the underlying spacetime manifold becomes noncommutative in nature. We note that it was not the case for Matsuo and Shibusa analysis in the presence of just the minimal length as has been reported in [34]. Finally we note that this study can be simply generalized to the supersymmetric quantum field theory.

\section{Acknowledgment}

K. Nozari work is financially supported by the Research Deputy of the Islamic Azad University, Sari Branch, Sari, Iran.

\section{References}

[1] G. Amelino-Camelia, M. Arzano, Y. Ling, and G. Mandanici, "Black-hole thermodynamics with modified dispersion relations and generalized uncertainty principles," Classical and Quantum Gravity, vol. 23, no. 7, pp. 2585-2606, 2006. 
[2] G. Veneziano, "A stringy nature needs just two constants," Europhysics Letters, vol. 2, p. 199, 1986.

[3] D. Amati, M. Cialfaloni, and G. Veneziano, "Superstring collisions at planckian energies," Physics Letters B, vol. 197, pp. 81-88, 1987.

[4] D. Amati, M. Cialfaloni, and G. Veneziano, "Can spacetime be probed below the string size?" Physics Letters B, vol. 216, pp. 41-47, 1989.

[5] D. J. Gross and P. F. Mende, "The high-energy behavior of string scattering amplitudes," Physics Letters B, vol. 197, pp. 129-134, 1987.

[6] K. Konishi, G. Paffuti, and P. Provero, "Minimum physical length and the generalized uncertainty principle in string theory," Physics Letters B, vol. 234, no. 3, pp. 276-284, 1990.

[7] R. Guida, K. Konishi, and P. Provero, "On the short distance behavior of string theories," Modern Physics Letters A, vol. 6, p. 1487, 1991.

[8] M. Kato, "Particle theories with minimum observable length and open string theory," Physics Letters B, vol. 245 , pp. 43-47, 1990.

[9] L. J. Garay, “Quantum gravity and minimum length," International Journal of Modern Physics A, vol. 10 , p. 145, 1995.

[10] S. Capozziello, G. Lambiase, and G. Scarpetta, “Generalized uncertainty principle from quantum geometry," International Journal of Theoretical Physics, vol. 39, no. 1, pp. 15-22, 2000.

[11] M. Maggiore, "A generalized uncertainty principle in quantum gravity ," Physics Letters B, vol. 304, pp. 65-69, 1993.

[12] M. Maggiore, "The algebraic structure of the generalized uncertainty principle," Physics Letters B, vol. 319, pp. 83-86, 1993.

[13] M. Maggiore, “Quantum groups, gravity, and the generalized uncertainty principle," Physical Review $D$, vol. 49, no. 10, pp. 5182-5187, 1994.

[14] S. Hossenfelder, "Can we measure structures to a precision better than the Planck length?" Classical and Quantum Gravity, vol. 29, Article ID 115011, 2012.

[15] P. Pedram, "New approach to nonperturbative quantum mechanics with minimal length uncertainty," Physical Review D, vol. 85, Article ID 024016, 2012.

[16] R. J. Adler, "Six easy roads to the Planck scale," American Journal of Physics, vol. 78, p. 925, 2010.

[17] A. Kempf, G. Mangano, and R. B. Mann, "Hilbert space representation of the minimal length uncertainty relation," Physical Review D, vol. 52, no. 2, pp. 1108-1118, 1995.

[18] F. Scardigli, "Generalized uncertainty principle in quantum gravity from micro-black hole gedanken experiment," Physics Letters B, vol. 452, pp. 39-44, 1999.

[19] G. Amelino-Camelia, "Relativity in spacetimes with short-distance structure governed by an observer-independent (planckian) length scale," International Journal of Modern Physics D, vol. 11, p. 35,2000 .

[20] G. Amelino-Camelia, "Relativity: special treatment," Nature, vol. 418, pp. 34-35, 2002.

[21] G. Amelino-Camelia, “Doubly-special relativity: first results and key open problems," International Journal of Modern Physics D, vol. 11, p. 1643, 2002.

[22] J. Kowalski-Glikman, "Introduction to doubly special relativity," Lecture Notes in Physics, vol. 669, pp. 131-159, 2005.

[23] G. Amelino-Camelia, J. Kowalski-Glikman, G. Mandanici, and A. Procaccini, "Phenomenology of doubly special relativity," International Journal of Modern Physics A, vol. 20, p. 6007, 2005.

[24] K. Imilkowska and J. Kowalski-Glikman, "Doubly special relativity as a limit of gravity," Lecture Notes in Physics, vol. 702, p. 279, 2006.

[25] J. Magueijo and L. Smolin, "Lorentz invariance with an invariant energy scale," Physical Review Letters, vol. 88, Article ID 1904303, 2002.

[26] J. Magueijo and L. Smolin, "Generalized Lorentz invariance with an invariant energy scale," Physical Review D, vol. 67, Article ID 044017, 2003.

[27] J. Magueijo and L. Smolin, "String theories with deformed energy-momentum relations, and a possible nontachyonic bosonic string," Physical Review D, vol. 71, Article ID 026010, 2005.

[28] J. L. Cortes and J. Gamboa, "Quantum uncertainty in doubly special relativity," Physical Review D, vol. 71, Article ID 065015, 2005.

[29] A. Kempf, "Quantum field theory with nonzero minimal uncertainties in positions and momenta," Journal of Mathematical Physics, vol. 38, Article ID 124014, p. 1374, 1997. 
[30] A. Kempf and G. Mangano, "Minimal length uncertainty relation and ultraviolet regularization," Physical Review D, vol. 55, Article ID 124014, p. 7909, 1997.

[31] A. Kempf, "Fields with finite information density ," Physical Review D, vol. 69, Article ID 124014, 2004.

[32] S. Hossenfelder, "Interpretation of quantum field theories with a minimal length scale," Physical Review D, vol. 73, Article ID 105013, 2006.

[33] S. Hossenfelder, "A note on quantum field theories with a minimal length scale," Classical and Quantum Gravity, vol. 25, Article ID 038003, 2008.

[34] T. Matsuo and Y. Shibusa, "Quantization of fields based on generalized uncertainty principle," Modern Physics Letters A, vol. 21, p. 1285, 2006.

[35] Y. Shibusa, "Supersymmetric field theory based on generalized uncertainty principle," International Journal of Modern Physics A, vol. 22, p. 5279, 2007.

[36] K. Nozari and A. Etemadi, "Minimal length, maximal momentum, and Hilbert space representation of quantum mechanics," Physical Review D, vol. 85, Article ID 104029, 2012.

[37] A. F. Ali, S. Das, and E. C. Vagenas, "Discreteness of space from the generalized uncertaintyprinciple," Physics Letters B, vol. 678, pp. 497-499, 2009.

[38] S. Das, E. C. Vagenas, and A. Farag Ali, "Discreteness of space from GUP II: relativistic wave equations," Physics Letters B, vol. 692, no. 5, p. 342, 2010.

[39] P. Pedram, K. Nozari, and S. H. Taheri, "The effects of minimal length and maximal momentum on the transition rate of ultra cold neutrons in gravitational field," Journal of High Energy Physics, vol. 3, article 93, 2011.

[40] J. D. Bjorken, "Applications of the chiral U(6) $\otimes U(6)$ algebra of current densities," Physical Review, vol. 148, p. $1467,1966$.

[41] K. Johnson and F. E. Low, "Current algebras in a simple model," Progress of Theoretical Physics, Supplement, no. 37-38, pp. 74-93, 1966.

[42] D. V. Ahluwalia, "Quantum measurement, gravitation, and locality," Physics Letters B, vol. 339, pp. 301-303, 1994.

[43] S. Doplicher, K. Fredenhagen, and J. E. Roberts, "Spacetime quantization induced by classical gravity," Physics Letters B, vol. 331, pp. 39-44, 1994.

[44] G. Amelino-Camelia, "Gravity-wave interferometers as probes of a low-energy effective quantum gravity," Physical Review D, vol. 62, Article ID 024015, 2000.

[45] G. Amelino-Camelia, D. Benedetti, F. D’Andrea, and A. Procaccini, "Comparison of relativity theories with observer-independent scales of both velocity and length/mass," Classical and Quantum Gravity, vol. 20, no. 24, pp. 5353-5370, 2003.

[46] D. McMahon, Quantum Field Theory Demystified, McGraw-Hill, 2008. 

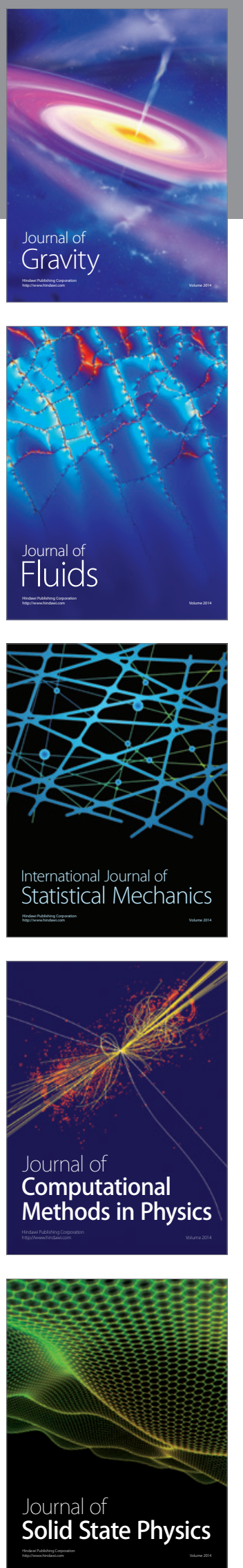

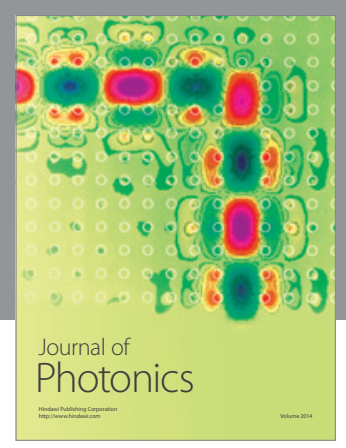

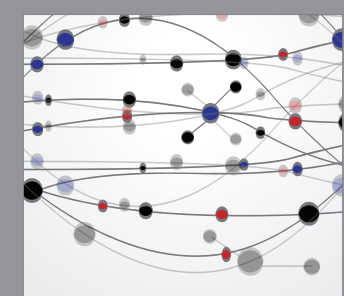

The Scientific World Journal
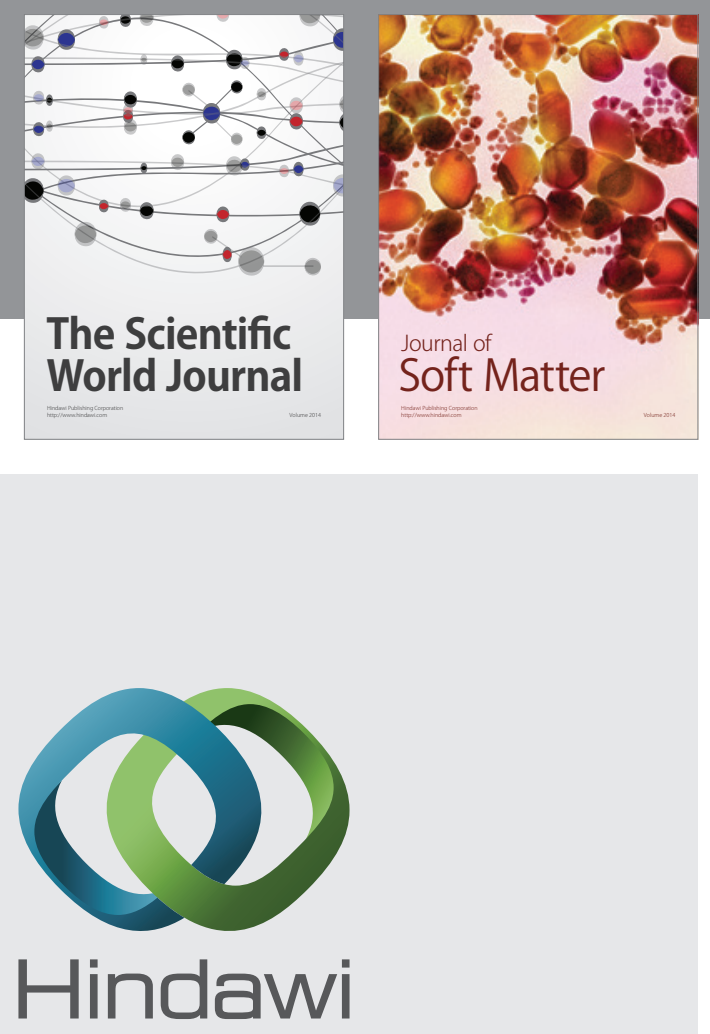

Submit your manuscripts at

http://www.hindawi.com
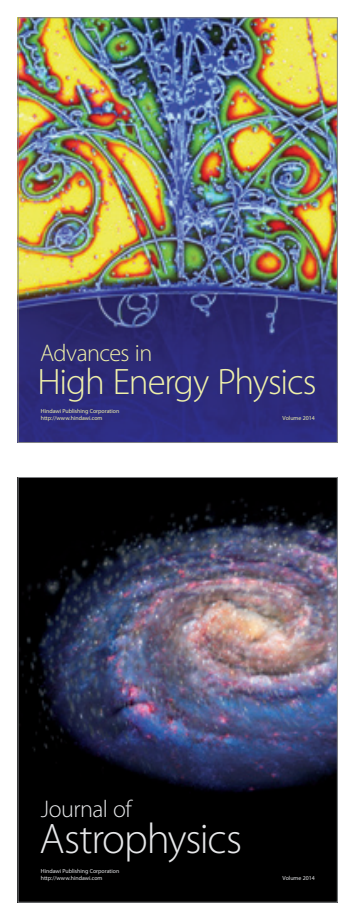
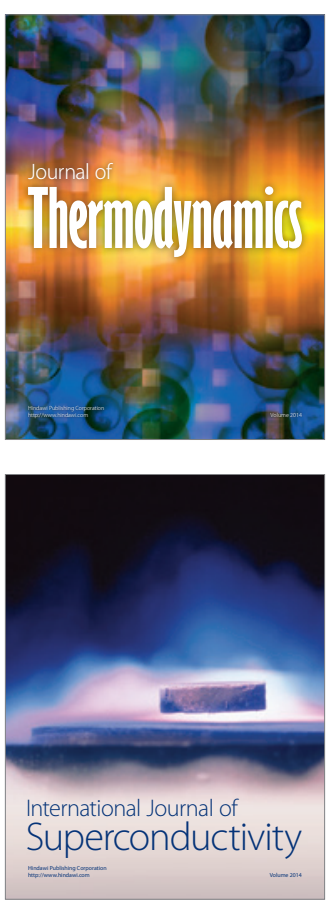
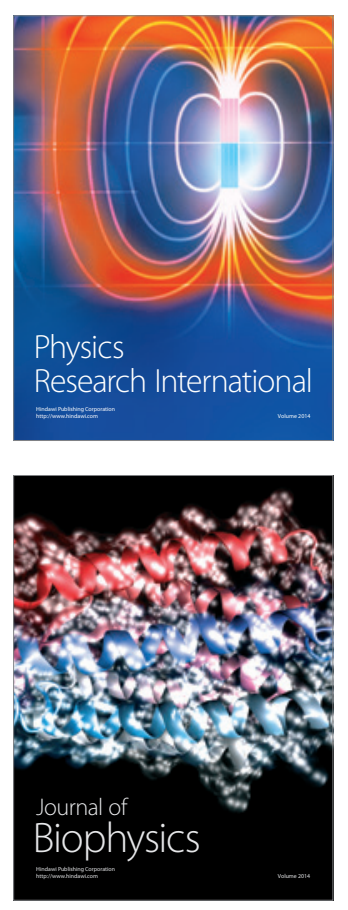
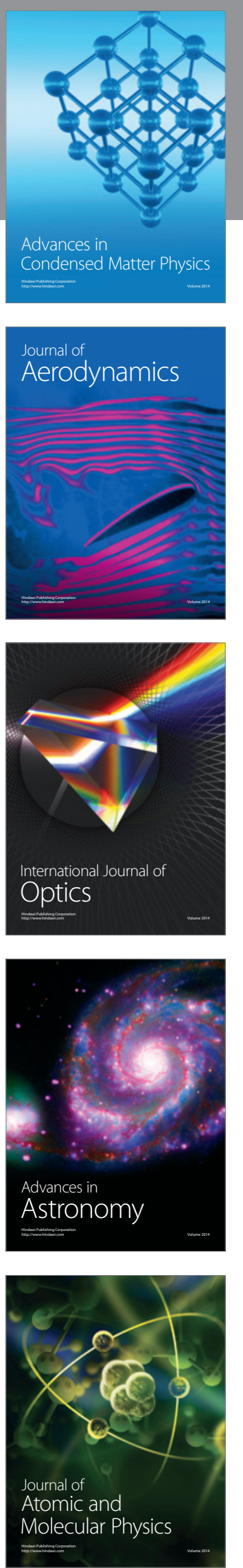